\title{
Vascular endothelial growth factor-A enhances indoleamine 2,3-dioxygenase expression by dendritic cells and subsequently impacts lymphocyte proliferation
}

\author{
Luciana Cavalheiro Marti ${ }^{1 /+}$, Lorena Pavon ${ }^{1,2}$, Patricia Severino', \\ Tatiana Sibov², Daiane Guilhen', Carlos Alberto Moreira-Filho', \\ ${ }^{1}$ Centro de Pesquisa Experimental, Instituto Israelita de Ensino e Pesquisa Albert Einstein \\ ${ }^{2}$ Centro de Pesquisa Experimental, Instituto do Cérebro, Hospital Israelita Albert Einstein, São Paulo, SP, Brasil \\ ${ }^{3}$ Departamento de Pediatria, Faculdade de Medicina, Universidade de São Paulo, São Paulo, SP, Brasil
}

\begin{abstract}
Dendritic cells (DCs) are antigen (Ag)-presenting cells that activate and stimulate effective immune responses by $T$ cells, but can also act as negative regulators of these responses and thus play important roles in immune regulation. Pro-angiogenic vascular endothelial growth factor (VEGF) has been shown to cause defective DC differentiation and maturation. Previous studies have demonstrated that the addition of VEGF to DC cultures renders these cells weak stimulators of Ag-specific T cells due to the inhibitory effects mediated by VEGF receptor 1 (VEGFRI) and/or VEGFR2 signalling. As the enzyme indoleamine 2,3-dioxygenase (IDO) is recognised as an important negative regulator of immune responses, this study aimed to investigate whether VEGF affects the expression of IDO by DCs and whether VEGF-matured DCs acquire a suppressor phenotype. Our results are the first to demonstrate that VEGF increases the expression and activity of IDO in DCs, which has a suppressive effect on Ag-specific and mitogen-stimulated lymphocyte proliferation. These mechanisms have broad implications for the study of immunological responses and tolerance under conditions as diverse as cancer, graft rejection and autoimmunity.
\end{abstract}

Key words: indoleamine-pyrrole 2,3-dioxygenase - dendritic cell -

vascular endothelial growth factor A - tolerance - immune - lymphocytes

Dendritic cells (DCs) are potent modulators of immune responses. In addition to their classical role as efficient antigen (Ag)-presenting cells and initiators of $\mathrm{T}$ cell responses, DCs can also suppress such responses and induce a state of immunological tolerance to Ags (Steinman 2012). It has been shown under several conditions that Ag presentation by DCs with an immature phenotype leads to either no response or tolerization to specific Ags, whereas Ag presentation by mature DC results in $\mathrm{T}$ cell proliferation and differentiation of effector functions. Because these cells only achieve full immunostimulatory capacity upon maturation, the selection of maturation factors becomes very important when DCs are derived in vitro (Steinman 2012).

Regarding maturation factors, prostaglandin E2 (PGE2), a lipid molecule released during inflammation, is typically the maturation factor of choice due to its ability to potentiate the effect of tumour necrosis factor-alpha (TNF- $\alpha$ ), stimulate interleukin (IL)-12 and increase chemokine receptor 7 expression (Rieser et al. 1997, Prechtel et al. 2007). Another factor that has been

doi: $10.1590 / 0074-0276130252$

Financial support: SBIBHIAE

+ Corresponding author: luciana.marti@einstein.br

Received 5 December 2012

Accepted 21 June 2013 used to mature DCs is Bacillus Calmette-Guérin (BCG), which acts through the activation of Toll-like receptors (TLRs) in a manner similar to lipopolysaccharide (LPS) (Tsuji et al. 2000).

However, certain molecules can also interfere with the efficiency of DC maturation, one of which is the proangiogenic vascular endothelial growth factor (VEGF), a soluble factor that is produced constitutively by nearly all types of solid tumours and stimulates both the growth of endothelial cells and new vessel formation. It has been shown that VEGF causes defective DC differentiation and maturation (Gabrilovich et al. 1998, Menetrier-Caux et al. 1998, Oyama et al. 1998, Zhang et al. 2003, Takahashi et al. 2004). Moreover, the addition of VEGF to DC cultures promotes a weak stimulus for Ag-specific T cells due to an inhibitory effect mediated by VEGF receptor 1 (VEGFR1)/Flt-1 signalling (Nemeth et al. 2004, Laxmanan et al. 2005). Of the described receptors for VEGF, VEGFR1 is the primary mediator of DC maturation inhibition (Dikov et al. 2005), whereas VEGFR2 is responsible for signal transduction in mature DCs, activating extracellular signal-regulated protein kinases 1 and 2 and impairing the DC stimulation of allogeneic lymphocytes (Kadambi et al. 2001, Mimura et al. 2007).

Another molecule involved in tolerogenesis is the enzyme indoleamine 2,3-dioxygenase (IDO), which is produced by many cell types, including DCs. The activation of IDO, mainly by interferon- $\gamma$, reduces the available concentration of its substrate tryptophan, which is essential for T cell proliferation (Mellor \& Munn 2004). However, independently of its activation, IDO can also 
act as a signal transducer in response to TGF- $\beta$ and confer a stable tolerogenic phenotype to plasmacytoid DCs, which in turn stimulate Tregs, thereby maintaining a state of tolerance (Chen 2011, Pallotta et al. 2011). A variety of conditions are finely tuned by IDO, ranging from pregnancy (Munn et al. 1998) and transplantation (Lan et al. 2010) to autoimmunity (Platten et al. 2005) and neoplasia (Katz et al. 2008).

Tumour microenvironments are naturally rich in immunomodulatory molecules that can interfere with DC maturation and prevent the development of effective anti-tumour responses (Lorusso \& Rüegg 2008). Therefore, it is important to identify the molecules and underlying mechanisms of a non-response, as they can also be important in other conditions, such as graft rejection and autoimmunity (Almand et al. 2000, Wood et al. 2010).

Although a role for VEGF in defective DC function has been suggested and immunological tolerance induction by IDO expression is well accepted, no studies have verified whether VEGF affects IDO expression levels or activity. Accordingly, in the present study, we investigated the effect of VEGF on IDO expression levels and its activity with regard to DC maturation and lymphocytic proliferation.

\section{MATERIALS AND METHODS}

Peripheral blood collection and $\mathrm{CD}^{+} 4^{+}$cell isolation - Peripheral blood was obtained from healthy volunteers after providing informed written consent according to the Ethical Research Committee or Israel Institute of Education and Research (105/02). Mononuclear cells were purified from the peripheral blood using density gradient Ficoll-Paque ${ }^{\mathrm{TM}}$ Plus (GE Healthcare, Buckinghamshire, UK) according the manufacturer's instructions. The $\mathrm{CD}_{1} 4^{+}$cell population was isolated from the mononuclear fraction by magnetic bead separation according to the manufacturer's protocol (Miltenyi Biotec, Bergisch Gladbach, Germany).

$D C$ generation and treatment with VEGF $A$ - The $\mathrm{CD}^{+} 4^{+}$cell population was dispensed into six-well plates containing X-vivo 15 medium (Cambrex, Walkersville, MD, USA) supplemented with antibiotic-antimycotics ${ }^{\mathrm{TM}}$ (Gibco, North Andover, MA, USA). To generate immature DCs, the cells were cultured in the presence of 20 $\mathrm{ng} / \mathrm{mL}$ recombinant IL-4 (rIL-4) (R\&D System) and 50 $\mathrm{ng} / \mathrm{ml} \mathrm{rGM-CSF} \mathrm{(R \& D} \mathrm{System)} \mathrm{for} \mathrm{six} \mathrm{days} \mathrm{(D6).} \mathrm{Ma-}$ ture DCs were obtained after $24 \mathrm{~h}$ (D7) of stimulation of the immature DC culture with $10 \mathrm{ng} / \mathrm{mL}$ rTNF- $\alpha$ (R\&D System) plus $0.01 \mathrm{mmol} / \mathrm{L}$ PGE2 (Sigma, St. Louis, MO, USA), referred to in this paper as PG-TNF; the concentrations used were previously described (Szabolcs et al. 2004). Alternatively, the immature DC cultures were stimulated to maturity by adding $20 \mu \mathrm{g}$ BCG (Ataulpho de Paiva Foundation, Rio de Janeiro, Brazil). The BCG suspension was previously titrated to obtain the lowest dose capable of stimulating efficient DC maturation (data not shown).

To study the effect of VEGF on DCs, the cells were cultivated after the initial day of culture in the presence or absence of $20 \mathrm{ng} / \mathrm{mL}$ of human rVEGF A, isoform 165 (Calbiochem-Millipore, Billerica, MA, USA), which it the predominant form of VEGF during angiogenesis.

Flow cytometry analysis - Staining of the cells for flow cytometry analyses was performed using commercially available monoclonal antibodies (mAbs) according to the manufacturer's instructions. Briefly, the cells were stained with the selected $\mathrm{mAbs}$ and incubated in the dark for $30 \mathrm{~min}$ at room temperature. The cells were then washed and fixed with $1 \%$ paraformaldehyde, with the exception of the cells tested for apoptosis, which were resuspended in the Annexin V binding buffer (BD Pharmingen, San Diego, CA, USA) provided with the reagent set. Intracellular staining was performed in previously fixed and permeabilised surface-stained cells prior to adding the labelled intracellular $\mathrm{mAb}$.

The mAbs used to evaluate DC markers or maturation were as follows: CD11c-PE clone:B-ly6, CD14fluorescein isothiocyanate (FITC) clone:M5E2, CD80PE clone:L307.4, CD83-PE clone:HB15e, CD86-PE clone:2331, CD123-PE clone:9F5 and CD209-PE clone:DCN46 (all purchased from BD Pharmingen); HLA-DR-PerCP-Cy5.5 clone:L243 and isotype controls (purchased from BD Biosciences, San Jose, CA, USA).

To assess lymphocyte proliferation, we used Ki-67FITC clone:B56 and CD3-PerCP Cy5.5 clone:SK7 in addition to isotype controls (BD Biosciences).

Monoclonal Abs against the VEGF receptors, including anti-VEGFR1-pure clone:49560 and VEGFR2PE clone:89106 (R\&D System) and VEGFR3-pure clone:9D9F9 (Chemicon, Temecula, CA, USA) and isotype controls were also used.

Rabbit anti-human IDO polyclonal antibody was purchased from Chemicon. Annexin V-FITC, propidium iodide, anti-caspase-3 active form-PE and isotype controls were purchased from BD Pharmingen. The secondary Abs included goat anti-mouse-FITC, goat anti-rabbitFITC and sheep anti-mouse-PE and were purchased from Chemicon.

The data were acquired using the FACSARIA flow cytometer (BD Biosciences) and the analyses were performed using the FACSDIVA software (BD Biosciences) and/or FLOWJO (Tree Star, Ashland, OR, USA).

Ag-specific proliferation assay - Mature DCs cultured in the presence or absence of VEGF $\left(10^{4}\right.$ cells $/ 100$ $\mu \mathrm{L})$ were co-cultivated in 96-well tissue culture plates (BD Biosciences-Discovery Labware, San Jose, CA, USA) for four days with autologous lymphocytes $\left(10^{5}\right.$ cells $/ 100 \mu \mathrm{L}$ ) previously stained with $5 \mu \mathrm{M} / \mathrm{mL}$ of carboxyfluorescein succinimidyl ester (CFSE) (Invitrogen). Ag-specific stimulation was performed by priming the DCs with lyophilised Candida albicans $(10 \mu \mathrm{g} / \mathrm{mL})$ and non-specific stimulation of the cultures was performed by adding the mitogen phytohaemagglutinin (PHA) 1 $\mu \mathrm{g} / \mathrm{mL}$ ). The DCs were $\gamma$-irradiated at 1,500 rads (GammaCell $1000{ }^{137} \mathrm{Cs}$ source) prior to co-cultivation.

After four days in culture, lymphocyte proliferation was monitored according to the CFSE fluorescence levels in the 530/30 channel. The lymphocyte populations were gated by size and granularity [side light scatter 
(SSC) vs. forward light scatter (FSC)] and the events selected in the lymphocyte gate were analysed in a second plot (histogram). The data analyses were performed using the FLOWJO software. The results were expressed using the division index, which represents the average number of divisions that a cell has undergone.

We also analysed non-specific (PHA) lymphocyte proliferation in co-cultures arranged as described, in which a specific inhibitor of IDO, 1-methyl-D-tryptophan (1-MT) at $1 \mathrm{mM}$ (Aldrich, St. Louis, MO, USA), was added at the beginning of the culture together with the VEGF-matured DCs.

After four days in culture, lymphocyte proliferation was monitored for the $\mathrm{CD}^{+}$population using KI67 nuclear staining as a proliferation marker. The lymphocytes were gated based on SSC vs. FSC, followed by $\mathrm{CD}^{+}$gating; the selected events were analysed in a second plot for KI-67 expression.

$R N A$ isolation and quantitative real-time polymerase chain reaction ( $q P C R$ ) - Monocytes, immature DCs and mature DCs were cultured in the presence or absence of VEGF for subsequent RNA isolation. Total RNA was isolated using the kit RNeasy ${ }^{\circledR}$ Mini Kit (Qiagen, Germany).

The extracted RNA was then reverse transcribed into cDNA using oligo, dNTPmix and reverse transcriptase from the SuperScript ${ }^{\mathbb{B}}$ II Reverse Transcriptase kit (Invitrogen). The primers were based on the Homo sapiens gene sequences for IDO and glyceraldehyde 3-phosphate dehydrogenase (GAPDH) obtained in the GenBank database (ncbi.nlm.nih.gov).

The primers were designed using Primer-select (DNAstar Inc, USA) and were synthesised by Invitrogen as IDO (forward, 5'-GGCAACCCCCAGCTATCAGA-3'; reverse, 5'-CAGGGAGACCAGAGCTTTCACA-3') and GAPDH(forward, 5'-GGAGAAGGCTGGGGCTCAT-3'; reverse, 5'-GTCCTTCCACGATACCAAAGTT-3'). The qPCR was performed using the Quanti Tect SYBR green PCR kit according to the manufacturer's instructions (Qiagen, Germany). The data were acquired and analysed using an ABI7500 sequence detection system. The qPCR data were analysed by normalising the cycle threshold $(\mathrm{Ct})$ values of the gene of interest to the $\mathrm{Ct}$ values of the housekeeping gene GAPDH (Livak \& Schmittgen 2001). The results are displayed showing monocytes as the baseline for comparison.

Ultrastructural characterisation by transmission electron microscopy (TEM) - The ultrastructures of monocytes and mature DCs grown in presence or absence of VEGF was examined by TEM.

The pellets of these cells were fixed in $0.2 \mathrm{M}$ cacodylate buffer with $1 \%$ glutaraldehyde for $2 \mathrm{~h}$ at $48^{\circ} \mathrm{C}$. The cells were then washed in cacodylate buffer twice for 15 min each. Post-fixation was performed with $1 \%$ osmium tetroxide for $1 \mathrm{~h}$ at $48^{\circ} \mathrm{C}$, followed by washing for $15 \mathrm{~min}$ in the same buffer.

For contrast, the pellet was immersed in a solution of uranyl acetate for $30 \mathrm{~min}$. After dehydration, the material was embedded in Epon resin diluted in acetone (1:1) and incubated at $48^{\circ} \mathrm{C}$ for $24 \mathrm{~h}$ with agitation.
The pellet was then transferred to pure Epon resin and incubated at $60^{\circ} \mathrm{C}$ for $72 \mathrm{~h}$ until completely polymerised. Semi and ultrathin sections were obtained with a Porter Blum ultramicrotome.

The semithin sections were stained with Azure II $(1 \%)$ and methylene blue (1\%). The ultrathin sections were placed on copper grids and stained with uranyl acetate and citrate. The grids were observed and photographed using TEM (Philips CM100).

Statistical analysis - Statistical analyses were performed by employing a two-tailed Student $t$ test, with statistical significance set at a $p$-value $\leq 0.05$. The Bonferroni correction was used when necessary.

\section{RESULTS}

$D C$ phenotypes after maturation induced $P G-T N F$ or $B C G$ - The differentiation of monocytes into DCs was assessed according to the expression of markers on D6 of culture in comparison to that of the original monocyte population at the start of the culture (D0).

The expression of maturation markers was determined one day after the maturation stimuli were added to the sixday-old cultures (D7). The frequencies of $\mathrm{CD}_{14}^{+}$populations on D0, D6 and D7 are shown in Fig. 1. The starting monocyte-enriched population was $82 \% \mathrm{CD}^{4}{ }^{+}$(Fig. 1A) (D0). After six days of culture in the presence of IL-4 + GM-CSF, the frequency of $\mathrm{CD} 14^{+}$cells had markedly decreased (Fig. 1A) (D6) and was maintained at low levels in either the PG + TNF or BCG-matured DCs (D7) (Fig. 1A). In contrast, CD209, a marker exclusively expressed on DCs (Geijtenbeek et al. 2000), was not observed on D0, but was found in nearly $90 \%$ of the cells on D6 and its frequency was maintained in the matured DCs (Fig. 1B). No differences in the frequency of cells expressing CD11c or HLA-DR were detected when comparing the cells at the D0, D6 and D7 stages of culture (Fig. 1C, D); however, the mean fluorescence index (MFI) showed increases on the order of two-three-fold. Significant increases in the frequency of $\mathrm{CD}_{123^{+}}$cells were also observed as the monocytes differentiated into DCs and subsequently matured (Fig. 1E) (D0 vs. D6 vs. D7). Taken together, these results indicate that the phenotypic differentiation of monocytes into DCs occurred in vitro.

DC maturation was evidenced by increases in the frequency and expression of the co-stimulation molecule CD80 and the maturation marker CD83 from D0-D6 and D7. In contrast, the MFI value of the co-stimulation molecule CD86 increased from D0-D6 and further increased as the cells matured (D7), although the CD86 $6^{+}$cell frequencies were maintained from D0-D7 (Fig. 1F-H). In general, treatment with $\mathrm{PG}+\mathrm{TNF} \alpha$ was more effective at promoting the maturation of DCs than $\mathrm{BCG}$, as the MFIs and frequencies of cells expressing CD80, CD83 and CD86 were lower after BCG treatment (Fig. 1F-H).

We also examined the monocytes and DCs for the presence of VEGFR and found that $90 \%$ of the monocytes expressed VEGFR1, VEGFR2 or VEGFR3 (Fig. $2 \mathrm{~A}-\mathrm{C}$ ), However, among the differentiated DCs (D6), the frequency of cells positive for each receptor fell to approximately $30 \%$, as did the VEGFR1 and VEGFR3 

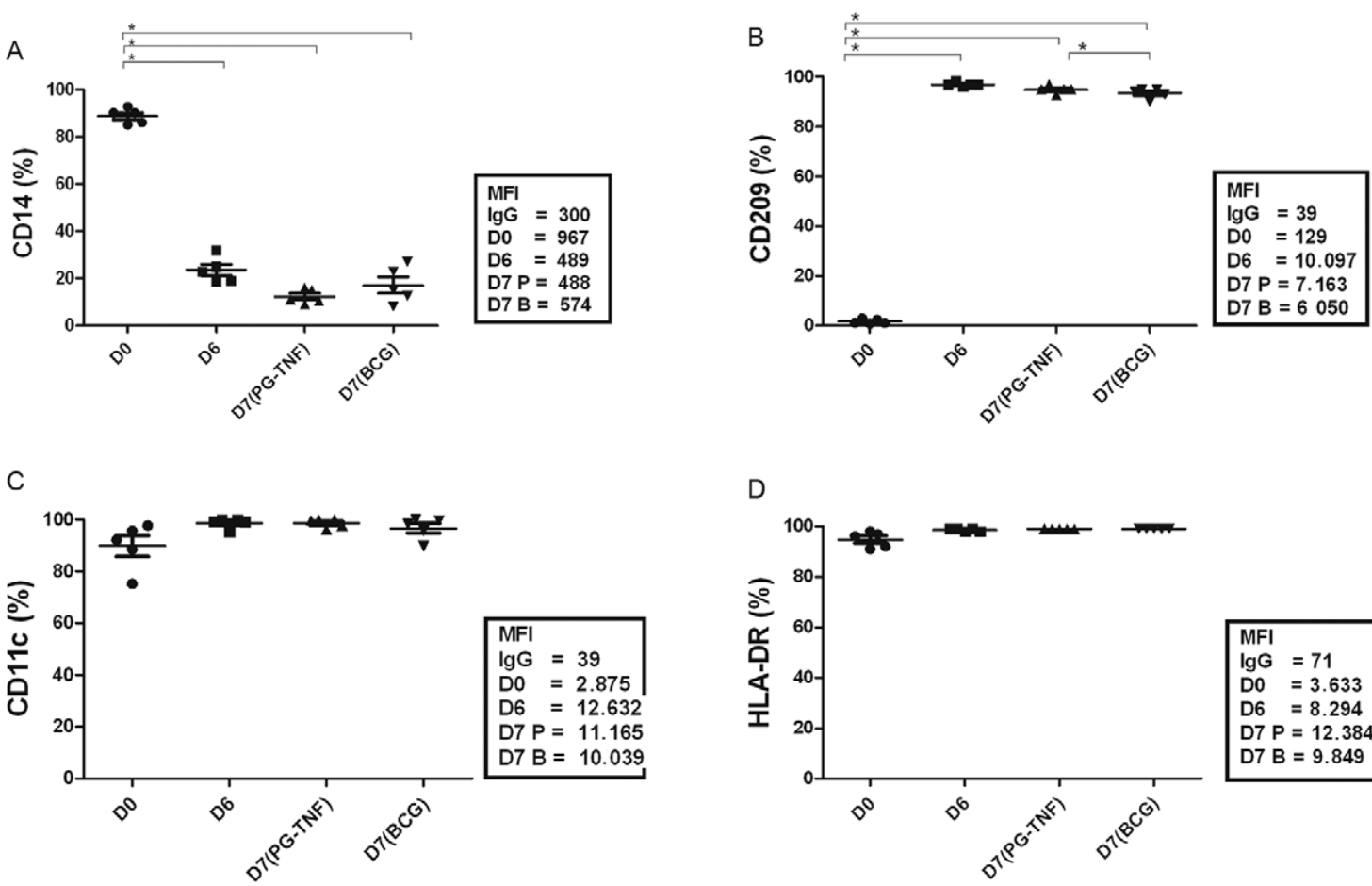

。
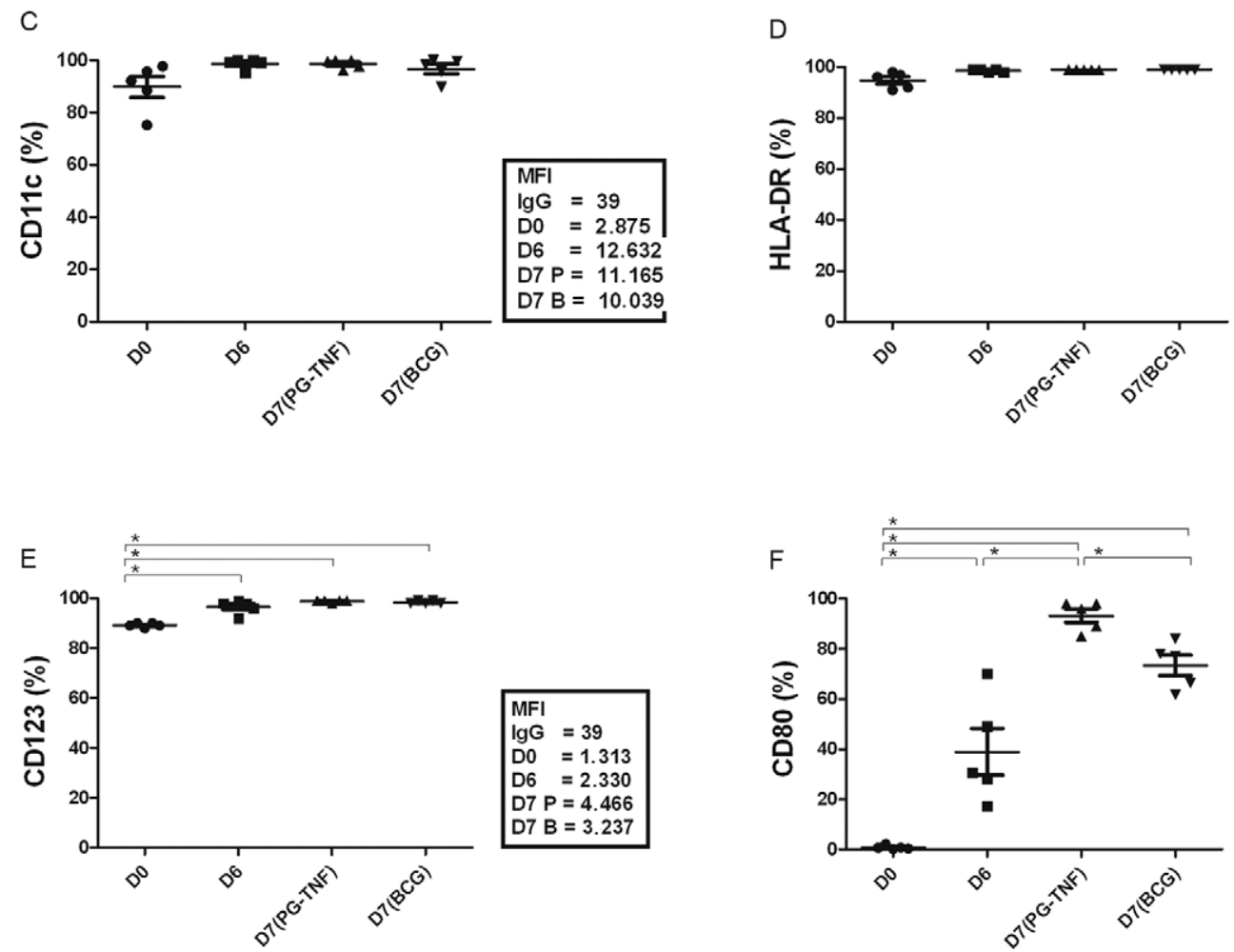

MFI

$\lg G=39$

$\mathrm{DO}=48$

$\mathrm{D} 6=575$

D7 $P=5.301$ D7 $B=3.237$

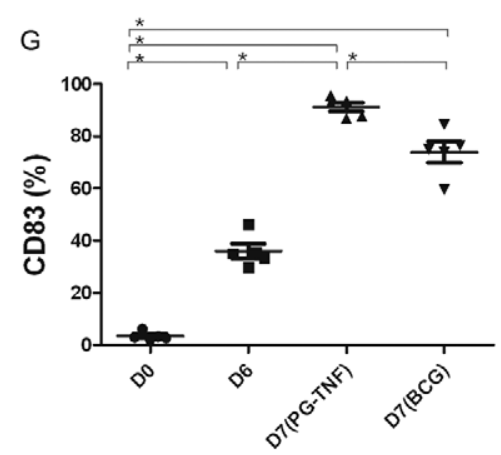

MFI

$\operatorname{IgG}=39$

$\mathrm{DO}=183$

D6 $=408$

$\mathrm{D} 7 \mathrm{P}=1.916$

D7 $B=825$

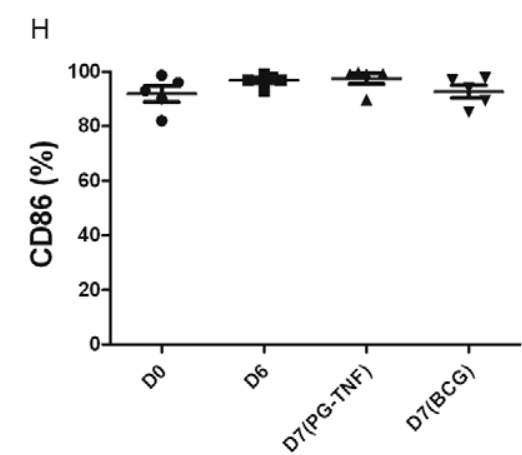

Fig. 1A-H: expression of cell surface markers by human monocytes differentiated in vitro to dendritic cells (DCs) and cultured in the presence of prostaglandin E2 + tumour necrosis factor alpha (PG-TNF) or Bacillus Calmette-Guérin (BCG) as maturation factors. Blood monocytes were isolated from peripheral blood mononuclear cells obtained from normal donors and cultured, day zero (D0), in the presence of rGM-CSF and recombinant interleukin four-six days to differentiate them to immature DC (D6) and further cultured for $24 \mathrm{~h}$ in PG-TNF or BCG (D7) as maturation factors. The cell suspensions were initially sorted by side light scatter (SSC) vs. forward light scatter (FSC) to exclude debris. The means and standard deviation (of 5 independent experiments) of the frequencies of positive cells for the different markers are shown in the graphs and significant $(\mathrm{p}<0.05)$ differences are indicated by an asterisk. The respective mean fluorescence intensities (MFI) for each fluorescent marker are shown as insets to each graph accompanied by its isotype-matched irrelevant fluorescent IgG control. 
MFI (but not for VEGFR2). At $24 \mathrm{~h}$ after the addition of maturation stimuli, the mean frequency of cells expressing VEGFR1 increased to $50 \%$, but this increase was only significant for PG + TNF and not for BCG. This result was most likely due to the greater intensity of the PG + TNF maturation stimulus and therefore its effect on the cells could be better observed and evaluated (Fig. 2A).

VEGF stimulates IDO expression by DCs treated with $P G+T N F$ - PG has been broadly used as a potent DC maturation factor and has been shown to induce IDO expression (Braun et al. 2005). IDO mRNA expression in monocytes is very low and the mean value was used as a baseline for the comparison of the values after differentiation and maturation.

As the monocytes differentiated into immature DCs (D6), the IDO relative mRNA expression levels were not significantly altered (Fig. 3A). However, a 1,000fold increase in IDO mRNA expression was observed in DCs that had been matured with PG + TNF for $24 \mathrm{~h}$ (Fig. 3A) and the presence of VEGF in the maturation cultures further increased IDO mRNA expression (Fig. $3 \mathrm{~A})$. This increase is best observed in Fig. 3B, in which the relative increases are presented on a linear scale. Indeed, we found that VEGF stimulated $100 \%$ increases in IDO mRNA expression in mature DCs (D7 V vs. D7). When these cell populations were stained for IDO and analysed by flow cytometry, the frequency of the cells matured with VEGF was 30\% higher than those cultivated without VEGF (Fig. 3C).

$V E G F$ influence on the morphology of $P G+T N F$ treated DCs, as revealed by TEM - Under TEM observation, the preparations of monocytes (D0) showed a predominance of regular, round-shaped cells containing ovoid nuclei with loose chromatin and evident nucleoli (Fig. 4A). The mature DCs that had been treated with PG + TNF were irregular and often star-shaped, exhibiting characteristic dendritic-like prolongments (Fig. 4B, C). These cells also demonstrated ovoid-shaped nuclei with loose chromatin and nucleoli; in addition, the mitochondria and rough endoplasmic reticulum were well developed and few lysosomes were observed (Fig.
4B-D). The morphology of the DCs treated with VEGF (in addition to PG + TNF) was not much different than the above-described features, although some cells displayed intense cytoplasmic vacuolation (Fig. 4E, F) associated with rough endoplasmic reticulum vesiculation (Fig. 4H); these changes were compatible with the initial senescent cell population.

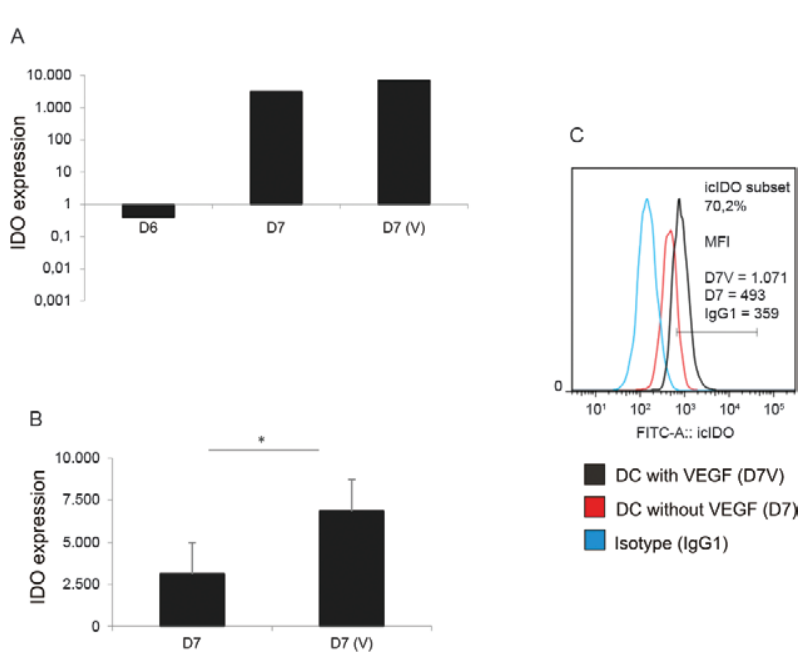

Fig. 3: effect of vascular endothelial growth factor (VEGF) during dendritic cell (DC) maturation on the relative expression of indoleamine 2,3-dioxygenase (IDO) mRNA and on intracellular IDO content detected by flow cytometry. Human peripheral blood monocytes were cultured and differentiated until day six (D6) as described in the legend of Fig. 1. Maturation was carried out with prostaglandin E2 + tumour necrosis factor alpha (PG-TNF) in the presence of VEGF: D7 (V) or just PG-TNF (D7). A: IDO mRNA relative expression in logarithmic scale; B: data in linear scale (asterisk means significant difference at $\mathrm{p}$ $<0.05$ ). For comparison, very low levels of IDO mRNA of immature DC (D6) compare to monocytes (baseline) and mature DC [D7: DC matured in the absence of VEGF; D7V: DC matured in the presence of VEGF (representative of 3 independent experiments)] are shown in A. The difference between D7V-D7 is better seem in B due to linear scale; C: flow cytometry data of mature DC stained for intracellular IDO accompanied by the mean fluorescence intensities (MFI) values; IgG1: fluorescein isothiocyanate-labelled isotype control.
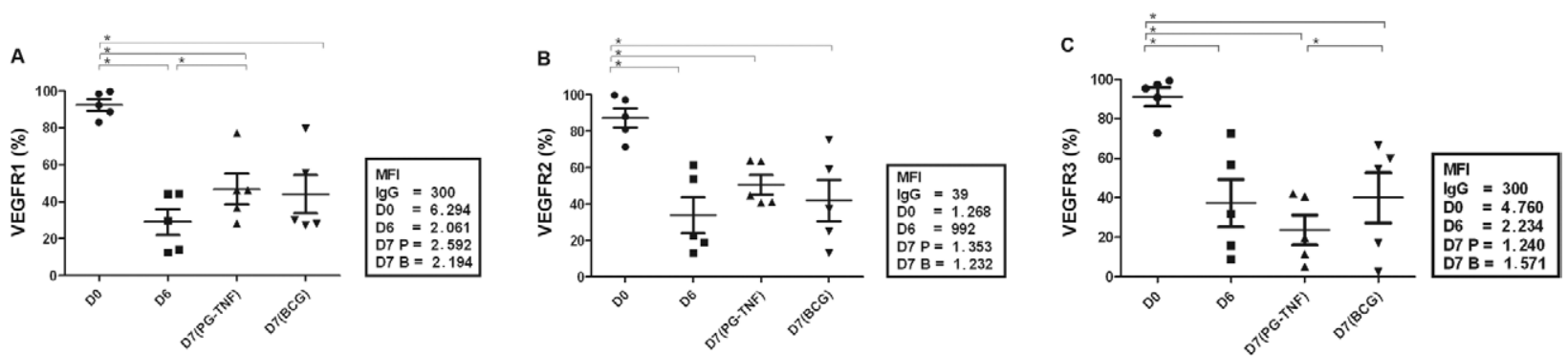

Fig. 2: expression of vascular endothelial growth factor (VEGF) receptors during human dendritic cell differentiation and subsequent maturation in the presence of prostaglandin E2 + tumour necrosis factor alpha (PG-TNF) or Bacillus Calmette-Guérin (BCG). Human peripheral blood monocytes were cultured and differentiated as described in the legend to Fig. 1. The mean frequencies and standard deviation ( $\mathrm{n}=5 \mathrm{independent}$ experiments) of dendritic cell expressing VEGFR1 (A), VEGFR2 (B) or VEGFR3 (C) are shown in the graphs accompanied by the mean values of mean fluorescence intensities (MFI). Significant differences $(\mathrm{p}<0.05)$ are indicated by an asterisk. 
VEGF effect on apoptosis in $P G+T N F$-treated DCs, as revealed by flow cytometry - The cell cultures matured with PG + TNF + VEGF showed an increased frequency of Annexin $\mathrm{V}+$ cells (a sign of early apoptosis) by $24 \mathrm{~h}$ (DC7V) and $48 \mathrm{~h}(\mathrm{DC} 8 \mathrm{~V})$ in comparison to cells cultured in the absence of VEGF (Fig. 5A). In addition, late apoptosis or necrosis was also evidenced in the VEGFtreated cultures after $48 \mathrm{~h}$, as shown by higher frequencies of double staining for Annexin and PI (DC8V) (Fig. $5 \mathrm{~B}, \mathrm{C})$. These data were also confirmed for late apoptosis, as a higher frequency of cells were positive for active caspase-3 (DC8V) (Fig. 5D). These results suggest that VEGF enhanced the apoptosis of DCs when present during the maturation process and were in agreement with the ultrastructural changes described for these cell cultures.

DCs exposed to VEGF during maturation are less capable of inducing Ag-specific or mitogen-triggered lymphocyte proliferation - To determine whether the changes
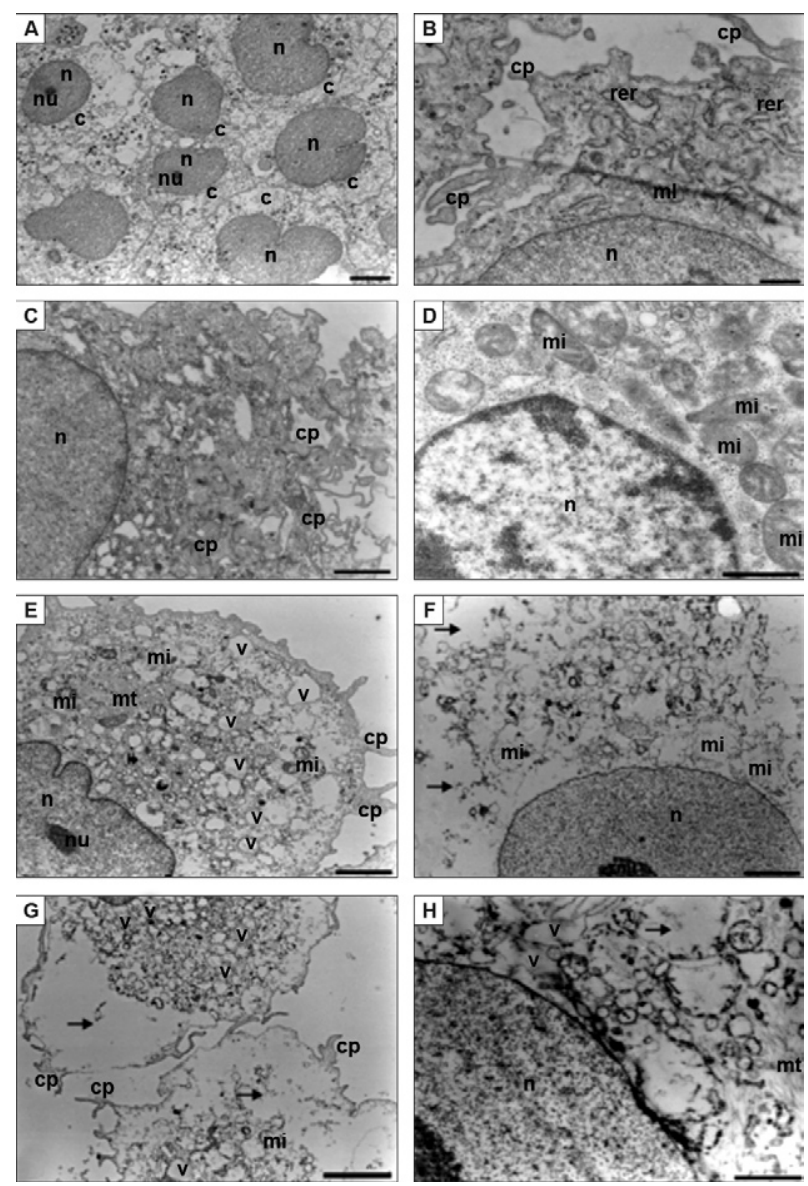

Fig. 4: transmission electron micrographs of monocytes and of dendritic cells (DCs) matured with prostaglandin E2 + tumour necrosis factor alpha (PG-TNF) in the presence or absence of vascular endothelial growth factor (VEGF). Monocytes and mature DCs were obtained as described in the legend of Fig. 3. A: monocyte culture on day zero; B-D: DCs matured in PG-TNF (D7); E-H: DCs matured in PG-TNF + VEGF (D7V); c: cytoplasm; cp: cytoplasmic prolongations; li: lysossome; mi: mitochondria; mt: microtubule; n: nuclei; nu: nucleoli; rer: rough endoplasmic reticulum; v: vacuolation. observed in VEGF-exposed DCs (e.g., IDO over-expression and accelerated apoptosis/senescence) would also translate into functional alterations, we next assessed Agspecific and PHA-triggered lymphocyte proliferation.

To this end, we employed a $2 \times 2$ factorial design in which we examined the level of autologous lymphocyte proliferation in the presence of DCs treated or not with VEGF. We used two independent stimuli, a specific stimulus dependent on presentation (C. albicans) and a non-specific mitogen, PHA, for four days of culture. We also repeated the experiment with PHA, evaluating lymphocyte proliferation by Ki-67 expression. To assess the effect of IDO on the maturation step, the DCs were matured in the presence of PG + TNF + VEGF and the IDO-inhibitor 1-MT was simultaneously added to the cultures; the resulting DC population was then tested in proliferation assays.

The VEGF-treated DCs caused a reduction in lymphocyte proliferation in response to Candida (Fig. 6A-C) and PHA (Fig. 6D-F) in comparison to the DCs matured in the absence of VEGF. This result was further confirmed by a reduction in lymphocyte proliferation to PHA according to Ki-67 expression (Fig. 7D, E). Of note, the addition of 1-MT to VEGF-treated DCs generated DCs that were as affective as DCs matured without VEGF (Fig. 7D, F), indicating that the impaired proliferation observed in the co-cultures of VEGF-treated DCs and lymphocytes was related to the activation of the IDO pathway during the maturation phase.

\section{DISCUSSION}

Although it is well known that VEGF affects DC maturation, our results demonstrate for the first time that VEGF increases the expression and activity of IDO, which has a suppressive effect on Ag-specific and mitogen-stimulated lymphocyte proliferation.

BCG was originally used as a maturation factor because of its similarity to LPS and because it was already in use as a human therapy. However, we observed that PG + TNF induced a more robust phenotype than BCG in DC maturation; thus, we used only PG + TNF in these experiments. Because we did not use BCG in the ensuing steps, these evaluations are valid for inflammatory environments, but are not necessarily relevant to infectious pathways.

PGE2 has been shown to induce IDO expression (Munn et al. 1998); furthermore, TNF- $\alpha$ in combination with PG induces IDO activity during DC maturation (Trinchieri 1995, Braun et al. 2005). IDO-expressing DCs are considered to be immunological tolerance inducers because they suppress lymphocyte activation and proliferation (Munn et al. 1998). Although PGE2 induces IDO mRNA expression through its receptor Eprostanoid 2 (EP2), enzyme activation is dependent on a second signal provided by TNFR or by TLR ligands (Braun et al. 2005). In contrast to these findings, it was reported that the induction of IDO expression by PGE2 occurs through its receptor EP4 instead of EP2 and that PGE2-matured DCs were more capable of inducing both allogeneic and Ag-specific $\mathrm{T}$ cell proliferation when 
A

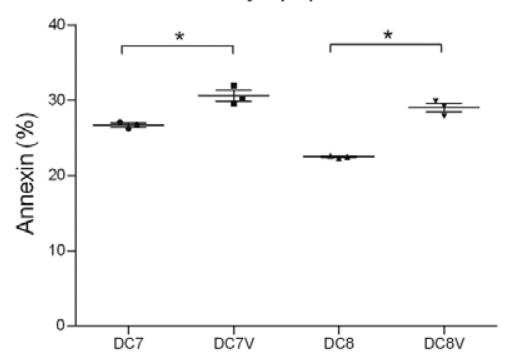

C

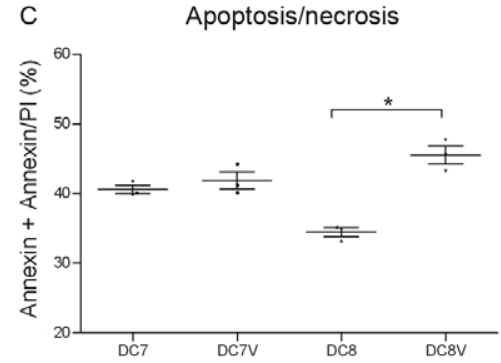

B

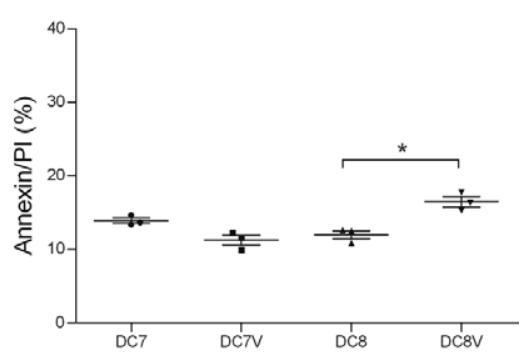

D

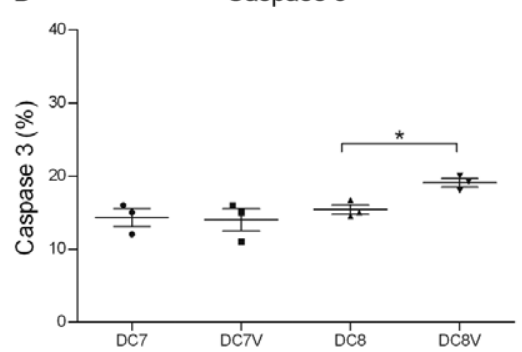

Fig. 5: effect of vascular endothelial growth factor (VEGF) on the expression of early and late markers of apoptosis and/or necrosis by dendritic cell (DC) cultures. The cells were cultured as described in legend of Fig. 3 with the maturation step carried out for $24 \mathrm{~h}$ with prostaglandin E2 + tumour necrosis factor alpha in the presence (DC7V) of VEGF or in its absence (DC7). In additional cultures the maturation step was carried out for $48 \mathrm{~h}$ in similar conditions, identified respectively as DC8V and DC8. The cells were double-stained for Annexin V/propidium iodide (apoptosis and/or necrosis) or stained only for caspase-3. A: cells that stained for Annexin V only (early apoptosis); B: cells double stained for Annexin V and propidium iodide only (late apoptosis/necrosis); C: frequencies of cells staining for Annexin V/PI + Annexin indicating the total number of cells undergoing apoptosis (early and late) and necrosis; D: cells expressing caspase 3 active (late apoptosis).

A

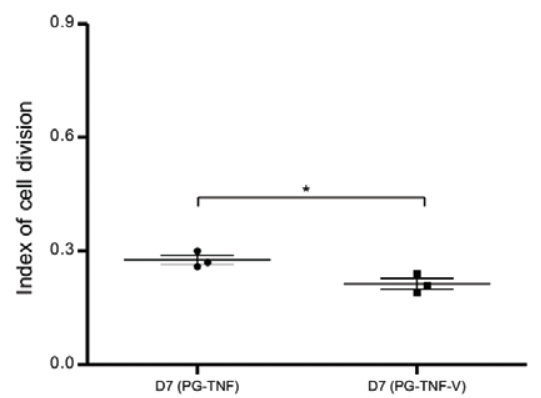

$\mathrm{D}$

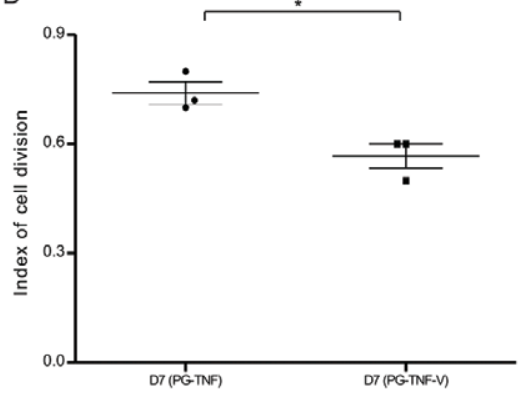

B
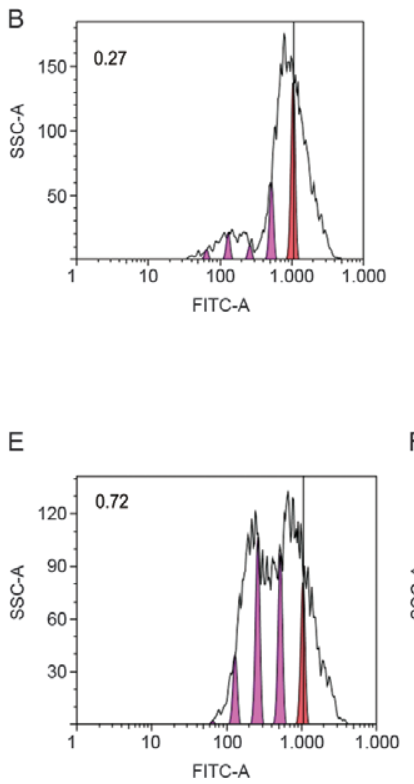

C
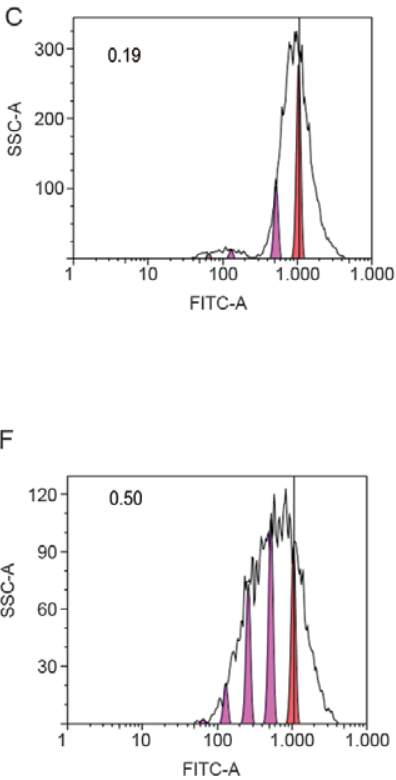

Fig. 6: T cell proliferation is impaired in the presence of dendritic cells (DCs) matured in the presence of vascular endothelial growth factor (VEGF) lymphocytes were co-cultivated with autologous DC that were treated with VEGF in addition to prostaglandin E2 + tumour necrosis factor alpha (PG-TNF) or only with PG-TNF during their maturation phase. Candida albicans was used to stimulate antigen-specific T lymphocyte proliferation (A-C). Histograms for lymphocytes labelled with side light scatter (SSC) vs. forward light scatter (FSC) are shown in B for co-cultures with PG-TNF-DC and in C for co-cultures with PG-TNF-V-DC. A: respective calculated indexes of cell division; D: respective calculated indexes of cell division for phytohemagglutinin (PHA)-stimulated cultures; D-F: PHA was used as T-cell mitogen; E, F: histograms of CFS-labelled lymphocytes co-cultured with DC matured without (E) or with VEGF (F). Asterisk means significant difference at $\mathrm{p}<0.05$. 
compared to DCs matured in the absence of this molecule (Krause et al. 2007). More recently (Lanzinger et al. 2012), it was shown that the stimulatory effect of PGE2-matured DCs on allogeneic $\mathrm{T}$ cells is variable and may be highly context dependent. When the IDO activity in the microenvironment is low, DCs act as effective stimulators of immune responses; however, once the enzymatic activity of IDO predominates, these cells suppress $\mathrm{T}$ cell responsiveness and/or promote regulatory $\mathrm{T}$ cell responses.

Because of the importance of IDO as a regulator of immune responses and the ubiquitous presence of VEGF in tumoural or inflammatory microenvironments, it was of interest to investigate whether VEGF would affect
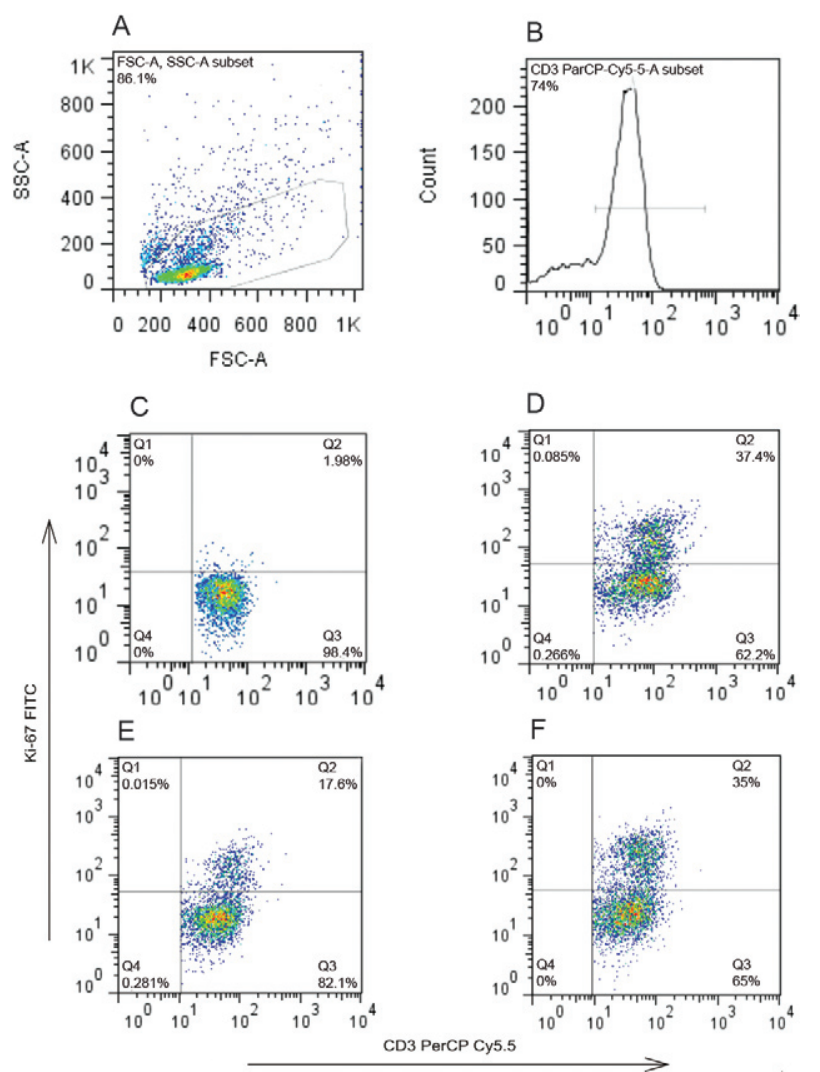

Fig. 7: addition of the inhibitor 1-methyl-D-tryptophan (1-MT) of indoleamine 2,3-dioxygenase (IDO) prevents vascular endothelial growth factor (VEGF)-matured dendritic cell (DC) from suppressing lymphocyte proliferation. $\mathrm{T}$ cell proliferation stimulated by phytohemagglutinin (PHA) was estimated by flow-cytometry of $\mathrm{CD}^{+}$lymphocytes expressing the antigen KI-67 (A) lymphocytes gated on side light scatter (SSC) vs. forward light scatter (FSC) (B), histogram of gated $\mathrm{CD}^{+}$cells (C), KI-67 expression on $\mathrm{CD}^{+}$lymphocytes without stimuli (1.56\% of the cells express the marker) (D), KI-67 expression on PHA-stimulated $\mathrm{CD}^{+}$lymphocytes co-cultured with autologous DC matured without VEGF (frequency of positive cells - 37.4\%) (E), KI-67 expression on PHA-stimulated $\mathrm{CD}^{+}$lymphocytes co-cultured with autologous DC matured with VEGF (frequency of positive cells - 17.6\%) and KI-67 expression on PHA-stimulated $\mathrm{CD}^{+}$lymphocytes co-cultured with autologous DC matured with VEGF in the presence of 1-MT as inhibitor of IDO (frequency of positive cells - 35\%) (representative of 3 independent experiments) (F). FITC: fluorescein isothiocyanate.
IDO expression by DCs. Indeed, our results show that IDO expression by DCs was significantly increased in the presence of VEGF compared to untreated DCs. In fact, both PG-TNF and VEGF contributed to augment IDO expression, suggesting a synergistic effect. Corroborating these data, we also observed that the induction of DC maturation by PG + TNF $\alpha$ significantly increased the expression of VEGFR1, thereby facilitating synergic signalling by VEGF (Dikov et al. 2005).

Our data concerning the ultrastructure of DCs treated with VEGF revealed morphological changes, predominantly vacuolation, which were compatible with processes that ultimately lead to cell death. This was further confirmed by Annexin V/propidium iodide staining of VEGF-treated DCs, which is indicative of apoptosis/necrosis (Henics \& Wheatley 1999, Orabona et al. 2008). The accelerated apoptosis of VEGF-treated DCs was most likely caused by a combination of factors. First, as has been previously shown, PG can stimulate programmed cell death by inducing the pro-apoptotic protein Bax (Lalier et al. 2011); second, VEGF acting in synergy would most likely further enhance apoptosis. In addition, tryptophan is an amino acid that is essential to cell survival and IDO activation reduces its availability to the cell (Broker et al. 2005).

One important question, however, was how DCs exposed to VEGF would function as regulators of the immune response. We found that specific (C. albicans) and mitogen-induced (PHA) lymphocyte proliferation were reduced in the presence of VEGF-treated DCs. This effect was due to IDO activity, as confirmed by experiments in which the addition of a specific IDO inhibitor (1-MT) during the maturation of VEGF-DCs completely abolished their suppressive activity on lymphocyte proliferation.

Another mechanism by which DCs develop an immunosuppressive phenotype is the ingestion of apoptotic cells (da Costa et al. 2011). However, because the DCs added to the co-cultures were mature and also $\gamma$-irradiated, their phagocytic activity was much reduced; thus, it is unlikely that such a mechanism was important to the observed suppression of lymphocyte proliferation in our assays. In addition, phagocytosis of apoptotic cells was not observed in our TEM examination of sections of VEGF-matured DCs.

Taken together, our results suggest that VEGF plays a role in the complex process of immunological tolerance, as it can stimulate DCs to over-activate the IDO pathway. The ensuing tryptophan depletion leads to the inhibition of $\mathrm{T}$ cell activation and expansion (Grohmann \& Bronte 2010, Kushwah \& Hu 2010). In addition, DCs that express IDO can stimulate the cellular general control non-depressible 2 kinase-dependent stress response in naïve and mature $T$ cells and in functionally quiescent $\mathrm{T}$ regulatory cells, leading to active bystander suppression (Fallarino et al. 2006, Sharma et al. 2007).

These mechanisms have broad implications in the study of immunological responses and tolerance under conditions as diverse as cancer, graft rejection and autoimmunity. 


\section{REFERENCES}

Almand B, Resser JR, Lindman B, Nadaf S, Clark JI, Kwon ED, Carbone DP, Gabrilovich DI 2000. Clinical significance of defective dendritic cell differentiation in cancer. Clin Cancer Res 6: $1755-1766$

Braun D, Longman RS, Albert ML 2005. A two-step induction of indoleamine 2,3-dioxygenase (IDO) activity during dendritic-cell maturation. Blood 106: 2375-2381.

Broker LE, Kruyt FA, Giaccone G 2005. Cell death independent of caspases: a review. Clin Cancer Res 11: 3155-3162.

Chen W 2011. IDO: more than an enzyme. Nat Immunol 12: 809-811.

da Costa TB, Sardinha LR, Larocca R, Peron JP, Rizzo LV 2011. Allogeneic apoptotic thymocyte-stimulated dendritic cells expand functional regulatory T cells. Immunology 133: 123-132.

Dikov MM, Ohm JE, Ray N, Tchekneva EE, Burlison J, Moghanaki D, Nadaf S, Carbone DP 2005. Differential roles of vascular endothelial growth factor receptors 1 and 2 in dendritic cell differentiation. J Immunol 174: 215-222.

Fallarino F, Grohmann U, You S, McGrath BC, Cavener DR, Vacca C, Orabona C, Bianchi R, Belladonna ML, Volpi C, Santamaria P, Fioretti MC, Puccetti P 2006. The combined effects of tryptophan starvation and tryptophan catabolites down-regulate $\mathrm{T}$ cell receptor zeta-chain and induce a regulatory phenotype in naive $\mathrm{T}$ cells. J Immunol 176: 6752-6761.

Gabrilovich D, Ishida T, Oyama T, Ran S, Kravtsov V, Nadaf S, Carbone DP 1998. Vascular endothelial growth factor inhibits the development of dendritic cells and dramatically affects the differentiation of multiple hematopoietic lineages in vivo. Blood 92: 4150-4166.

Geijtenbeek TB, Torensma R, van Vliet SJ, van Duijnhoven GC, Adema GJ, van Kooyk Y, Figuredor CG 2000. Identification of DC-SIGN, a novel dendritic cell-specific ICAM-3 receptor that supports primary immune responses. Cell 100: 575-585.

Grohmann U, Bronte V 2010. Control of immune response by amino acid metabolism. Immunol Rev 236: 243-264.

Henics T, Wheatley DN 1999. Cytoplasmic vacuolation, adaptation and cell death: a view on new perspectives and features. Biol Cell 91: 485-498.

Kadambi A, Carreira CM, Yun CO, Padera TP, Dolmans DE, Carmeliet P, Fukumura D, Jain RK 2001. Vascular endothelial growth factor (VEGF)-C differentially affects tumor vascular function and leukocyte recruitment: role of VEGF-receptor 2 and host VEGF-A. Cancer Res 61: 2404-2408.

Katz JB, Muller AJ, Prendergast GC 2008. Indoleamine 2,3-dioxygenase in T-cell tolerance and tumoral immune escape. Immunol Rev 222: 206-221.

Krause P, Singer E, Darley PI, Klebensberger J, Groettrup M, Legler DF 2007. Prostaglandin E2 is a key factor for monocyte-derived dendritic cell maturation: enhanced $\mathrm{T}$ cell stimulatory capacity despite IDO. J Leukoc Biol 82: 1106-1114.

Kushwah R, Hu J 2010. Dendritic cell apoptosis: regulation of tolerance versus immunity. J Immunol 185: 795-802.

Lalier L, Pedelaborde F, Braud C, Menanteau J, Vallette FM, Olivier C 2011. Increase in intracellular PGE2 induces apoptosis in Baxexpressing colon cancer cell. BMC Cancer 11: 153.

Lan Z, Ge W, Arp J, Jiang J, Liu W, Gordon D, Healey D, de Benedette M, Nicolette C, Garcia B, Wang H 2010. Induction of kidney allograft tolerance by soluble CD83 associated with prevalence of tolerogenic dendritic cells and indoleamine 2,3-dioxygenase. Transplantation 90: 1286-1293.

Lanzinger M, Jürgens B, Hainz U, Dillinger B, Raberger J, Fuchs D, Heitger A 2012. Ambivalent effects of dendritic cells displaying prostaglandin E2-induced indoleamine 2,3-dioxygenase. Eur $J$ Immunol 42: 1117-1128.

Laxmanan S, Robertson SW, Wang E, Lau JS, Briscoe DM, Mukhopadhyay D 2005. Vascular endothelial growth factor impairs the functional ability of dendritic cells through Id pathways. Biochem Biophys Res Commun 334: 193-198.

Livak KJ, Schmittgen TD 2001. Analysis of relative gene expression data using real-time quantitative PCR and the 2(-Delta Delta C(T)) Method. Methods 25: 402-408.

Lorusso G, Rüegg C 2008. The tumor microenvironment and its contribution to tumor evolution toward metastasis. Histochem Cell Biol 130: 1091-1103.

Mellor AL, Munn DH 2004. IDO expression by dendritic cells: tolerance and tryptophan catabolism. Nat Rev Immunol 4: 762-774.

Menetrier-Caux C, Montmain G, Dieu MC, Bain C, Favrot MC, Caux C, Blay JY 1998. Inhibition of the differentiation of dendritic cells from $\mathrm{CD} 34(+)$ progenitors by tumor cells: role of interleukin- 6 and macrophage colony-stimulating factor. Blood 92: 4778-4791.

Mimura K, Kono K, Takahashi A, Kawaguchi Y, Fujii H 2007. Vascular endothelial growth factor inhibits the function of human mature dendritic cells mediated by VEGF receptor-2. Cancer Immunol Immunother 56: 761-770.

Munn DH, Zhou M, Attwood JT, Bondarev I, Conway SJ, Marshall B, Brown C, Mellor AL 1998. Prevention of allogeneic fetal rejection by tryptophan catabolism. Science 281: 1191-1193.

Nemeth ZH, Deitch EA, Davidson MT, Szabo C, Vizi ES, Hasko G 2004. Disruption of the actin cytoskeleton results in nuclear factor-kappaB activation and inflammatory mediator production in cultured human intestinal epithelial cells. J Cell Physiol 200: 71-81

Orabona C, Pallotta MT, Volpi C, Fallarino F, Vacca C, Bianchi R, Belladonna ML, Fioretti MC, Grohmann U, Puccetti P 2008. SOCS3 drives proteasomal degradation of indoleamine 2,3-dioxygenase (IDO) and antagonizes IDO-dependent tolerogenesis. Proc Natl Acad Sci 105: 20828-20833.

Oyama T, Ran S, Ishida T, Nadaf S, Kerr L, Carbone DP, Gabrilovich DI 1998. Vascular endothelial growth factor affects dendritic cell maturation through the inhibition of nuclear factor-kappa B activation in hemopoietic progenitor cells. J Immunol 160: 1224-1232.

Pallotta MT, Orabona C, Volpi C, Vacca C, Belladonna ML, Bianchi R, Servillo G, Brunacci C, Calvitti M, Bicciato S, Mazza EM, Boon L, Grassi F, Fioretti MC, Fallarino F, Puccetti P, Grohmann $\mathrm{U}$ 2011. Indoleamine 2,3-dioxygenase is a signaling protein in long-term tolerance by dendritic cells. Nat Immunol 12: 870-878.

Platten M, Ho PP, Youssef S, Fontoura P, Garren H, Hur EM, Gupta R, Lee LY, Kidd BA, Robinson WH, Sobel RA, Selley ML, Steinman L 2005. Treatment of autoimmune neuroinflammation with a synthetic tryptophan metabolite. Science 310: 850-855.

Prechtel AT, Turza NM, Theodoridis AA, Steinkasserer A 2007. CD83 knockdown in monocyte-derived dendritic cells by small interfering RNA leads to a diminished T cell stimulation. $J$ Immunol 178: 5454-5464.

Rieser C, Bock G, Klocker H, Bartsch G, Thurnher M 1997. Prostaglandin E2 and tumor necrosis factor alpha cooperate to activate human dendritic cells: synergistic activation of interleukin 12 production. J Exp Med 186: 1603-1608. 
Sharma MD, Baban B, Chandler P, Hou DY, Singh N, Yagita H, Azuma M, Blazar BR, Mellor AL, Munn DH 2007. Plasmacytoid dendritic cells from mouse tumor-draining lymph nodes directly activate mature Tregs via indoleamine 2,3-dioxygenase. J Clin Invest 117: 2570-2582.

Steinman RM 2012. Decisions about dendritic cells: past, present and future. Annu Rev Immunol 30: 1-22.

Szabolcs P, Park KD, Marti L, Deoliveria D, Lee YA, Colvin MO, Kurzberg J 2004. Superior depletion of alloreactive T cells from peripheral blood stem cell and umbilical cord blood grafts by the combined use of trimetrexate and interleukin-2 immunotoxin. Biol Blood Marrow Transplant 10: 772-783.

Takahashi A, Kono K, Ichihara F, Sugai H, Fujii H, Matsumoto Y 2004. Vascular endothelial growth factor inhibits maturation of dendritic cells induced by lipopolysaccharide, but not by proinflammatory cytokines. Cancer Immunol Immunother 53: 543-550.
Trinchieri G 1995. Interleukin-12: a proinflammatory cytokine with immunoregulatory functions that bridge innate resistance and antigen-specific adaptive immunity. Аппи Rev Immunol 13: 251-276.

Tsuji S, Matsumoto M, Takeuchi O, Akira S, Azuma I, Hayashi A, Toyoshima K, Seya T 2000. Maturation of human dendritic cells by cell wall skeleton of Mycobacterium bovis bacillus Calmette-Guérin: involvement of toll-like receptors. Infect Immun 68: 6883-6890.

Wood KJ, Bushell AR, Jones ND 2010. The discovery of immunological tolerance: now more than just a laboratory solution. J Immunol 184: 3-4.

Zhang L, Conejo-Garcia JR, Katsaros D, Gimotty PA, Massobrio M, Regnani G, Makrigiannakis A, Gray H, Schlienger K, Liebman MN, Rubin SC, Coukos G 2003. Intratumoral T cells, recurrence and survival in epithelial ovarian cancer. $N$ Engl J Med 348: 203-213. 